\title{
Bounds on a Gramian-Based Interaction Measure for Robust Control Structure Selection
}

\author{
Miguel Castaño Arranz ${ }^{\star 1}$, Wolfgang Birk ${ }^{1}$
}

\begin{abstract}
This paper reviews a method for approximating the bounds on a gramian-based Interaction Measure (IM) for systems described by uncertain parametric models. The considered IM is the Participation Matrix. The reviewed method is based on analyzing the uncertainty in the area enclosed by the Nyquist curve. Conditions for the exactitude of this method are derived, and implementation issues are discussed.

It is shown that the reviewed method is applicable to nonparametric uncertain models of the frequency response function, which enables the estimation of the indicator from process data including the uncertainty bounds.

Using these bounds, robust control structure selection can be performed.
\end{abstract}

\section{INTRODUCTION}

A critical step in the design of control loops in multivariable systems is the choice of the controller structure. This is done by selecting a subset of the most significant input-output modeled interconnections, which will form a reduced model on which the control design will be based.

Current methods for control structure selection include the gramian-based Interaction Measures (IMs), which quantify the significance of each of the input-output channels of a multivariable system by using different gramian-based system operators. The IM considered in this paper is the Participation Matrix (PM) [1], which uses the squared HilbertScmhmidt (HS) norm.

The IMs are traditionally evaluated using a nominal model of the process. However, all process models are affected by uncertainties as simplifications and approximations are unavoidable during modeling. Thus, the validity of the control structure suggested by the IMs cannot be assessed by only analyzing the nominal model.

A previous result in [2], introduces an inequality later used in [3] for the uncertain analysis of the PM. However, simulation examples showed evidence that this approach is likely to derive in very conservative results. To overcome this conservativeness, a preliminary method was proposed to obtain a numerical approximation of the bounds on the PM. The method is based on analyzing the uncertainty in the area enclosed by the Nyquist diagram, since this area is closely related to the value of the squared HS norm (see [4]). The fact that the latter method is an approximation of the analytical bounds in which no inequality is involved motivates its further development and study. The method had the weakness of presenting inaccurate results in simulations

\footnotetext{
* Corresponding author: miguel. castano@ltu.se.

${ }^{1}$ Control Engineering Group, Department of Computer science, Electrical and Space Engineering, Luleå University of Technology, SE-971 87 Luleå, Sweden.
}

with systems of high order and with large uncertainty. The aim of this paper is to explore the applied potential and weaknesses of this method, and to discuss implementation issues.

The needed models for applying this approach are usually derived from first principle equations with uncertain parameters [5], or from model error modeling [6]. However, parametric modeling is a time consuming task, and its complexity increases with the number of process variables.

An application of the considered method is here presented to compute bounds on the estimation of the PM. This estimation is performed in the frequency domain from a nonparametric model of the Frequency Response Function (FRF). This will allow an empirical robust selection of a set of the most significant input-output interconnections on which the controller will be based. In addition, in the case of requiring more sophisticated models for the controller synthesis, the modeling effort only needs to be placed on this set of significant interconnections.

The layout of the paper is as follows. The preliminaries in Section II include information on the PM and its relationship with the Nyquist diagram, as well as a description of how the considered model uncertainty influences the Nyquist diagram. Section III reviews the method described in [3] for approximating the bounds on the PM and discusses the cases in which this method yields significant inaccuracies. Section IV introduces an application of the method for obtaining a robust estimation of the squared HS norm from process data. The conclusions are derived in Section V.

\section{Preliminaries}

\section{A. System gramians}

Given a continuous time system represented by the quadruple $(\mathrm{A}, \mathrm{B}, \mathrm{C}, 0)$ in state-space representation, the controllability $(\mathrm{P})$ and observability $(\mathrm{Q})$ gramians are obtained by solving the following continuous-time Lyapunov equations [5]:

$$
A P+P A^{T}+B B^{T}=0 ; A^{T} Q+Q A+C^{T} C=0
$$

$P$ quantifies how hard it is to control the system states from the inputs, and $Q$ quantifies how hard it is to observe the process states from the outputs.

Since $P$ and $Q$ depend on the state space realization, the product $P Q$ is formed. $P Q$ is a matrix with nonnegative eigenvalues which are independent of the state space realization, and the sum of its eigenvalues equals its trace.

The trace of $P Q$ quantifies the combined abilities of the inputs and outputs to control and observe the process state, 
or in another words, it quantifies the connection of the input and output spaces via the state space.

\section{B. Participation Matrix (PM)}

The PM was introduced as [1]:

$$
\phi_{i j}=\frac{\operatorname{tr}\left(P_{j} Q_{i}\right)}{\sum_{\substack{i=1 \\ j=1}}^{m, n} \operatorname{tr}\left(P_{j} Q_{i}\right)}
$$

where $\operatorname{tr}\left(P_{j} Q_{i}\right)$ is the trace of the product of the controllability and observability gramians of the $(i, j)$ input-output channel.

The used normalization implies that all the elements in the PM add up to 1 , and the element $\phi_{i j}$ quantifies the relative contribution of the $(i, j)$ input-output channel in the process.

When the PM is used for control structure design, a subset of the input-output channels is selected as the most significant. A total contribution of the selected channels higher than 0.7 is expected to derive in a satisfactory performance.

The normalization in the computation of the PM involves that a change in the value $\operatorname{tr}\left(P_{j} Q_{i}\right)$ for an unique inputoutput channel may influence all the elements in the PM. To avoid this dependency, an index array containing the values $\operatorname{tr}\left(P_{j} Q_{i}\right)$ will be analyzed, and denoted by $\tilde{\phi}$ :

$$
\tilde{\phi}_{i j}=\operatorname{tr}\left(P_{j} Q_{i}\right)
$$

In this index array, as in its normalized version, the largest elements identify the most significant input-output channels.

\section{Relationship of $\operatorname{tr}(P Q)$ with the frequency domain.}

The indicator $\operatorname{tr}(\mathrm{PQ})$ equals the squared Hilbert-Schmidt (HS) norm. This comes from the fact that this norm is the square root of the sum of the squared singular values of the Hankel operator, and the squared nonzero singular values of the Hankel operator are equal to the eigenvalues of $P Q$.

$$
\left\|G_{i j}\right\|_{H S}^{2}=\operatorname{tr}\left(P_{j} Q_{i}\right)
$$

Besides, it was shown in [4] that the squared HS norm is equal to the area enclosed by the oriented Nyquist diagram divided by $\pi$, with oriented meaning clockwise direction:

$$
\operatorname{tr}\left(P_{j} Q_{i}\right)=\pi^{-1} A_{c}\left(\Gamma_{i j}(\omega)\right)
$$

where $A_{c}\left(\Gamma_{i j}(\omega)\right)$ is area enclosed by $\Gamma_{i j}(\omega)=G_{i j}(\mathbf{j} \omega)$ with $\omega$ evaluated from $-\infty$ to $\infty$ in continuous-time systems.

\section{Representing model uncertainty}

To represent model uncertainty in multiplicative form in a SISO system, the following notation will be used:

$\Pi$ : uncertainty set. Includes all the possible plants due to uncertainty.

$G(s) \in \Pi$ : nominal plant.

$G p(s) \in \Pi$ : particular perturbed plant.

The uncertainty set is then described by:

$$
\Pi: G p(s)=G(s)(1+W(s) \Delta(s)) ; \quad|\Delta(\mathbf{j} \omega)| \leq 1, \forall \omega
$$

$W(s)$ is an uncertainty weight designed to represent the uncertainty. $\Delta(s)$ is a random model perturbation, and represents any stable transfer function with magnitude less or

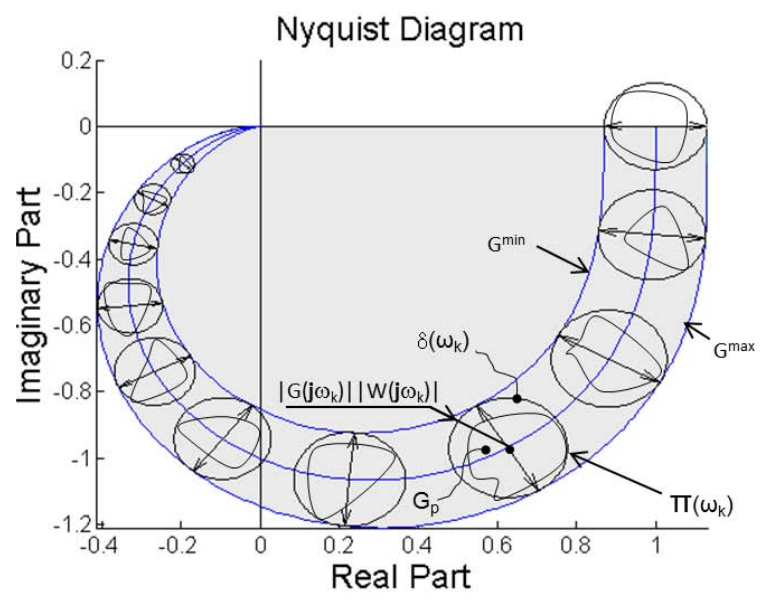

Fig. 1. Nyquist diagram perturbed by uncertainty.

equal than one at each frequency. $G(s)(1+W(s) \Delta(s))$ describes at each frequency $\omega_{k}$ a circular region of uncertainty centered in $G\left(\mathbf{j} \omega_{k}\right)$ with radius equal to $\left|G\left(\mathbf{j} \omega_{k}\right) \cdot W\left(\mathbf{j} \omega_{k}\right)\right|$. As illustrated in Fig. 1, $W(s)$ has to be selected so that the uncertainty set $\Pi$ includes the possible uncertainty at each frequency $\omega_{k}$. In the sequel, the family of circumferences which include the uncertainty set will be named as $\delta$ and can be parameterized as function of the frequency $\omega$ :

$$
\delta(\omega, \theta)=G(\mathbf{j} \omega)+|G(\mathbf{j} \omega) W(\mathbf{j} \omega)| \cdot e^{\mathbf{j} \theta} \quad ; \quad \theta \in[0,2 \pi)
$$

Usually uncertainty weights are designed as rational transfer functions, but other options are possible, like defining the weight as a real valued function of $\omega$ ([7]). For the results presented in this paper, the latter option is preferred, since it will involve in most cases less computational complexity.

Here the uncertainty weights $\mathrm{W}$ are required to have continuous first derivatives, besides the following typical condition for uncertainty weights:

$$
\lim _{\omega \rightarrow \infty} W \neq \pm \infty
$$

For MIMO systems, each of the input-output channels $G_{i j}(s)$ is assumed to be independently perturbed by multiplicative uncertainty.

\section{III. $\operatorname{tr}(P Q)$ FOR UNCERTAIN PARAMETRIC MODELS}

We describe in this section a procedure to approximate the uncertainty bounds on $\operatorname{tr}(P Q)$ from the uncertainty in the area enclosed by the Nyquist diagram and discuss its exactitude.

\section{A. Approximating the bounds of $\operatorname{tr}(P Q)$}

For a SISO model affected by multiplicative uncertainty as in Equation (1), the uncertainty area in the Nyquist diagram can be approximated by swiping along the Nyquist curve the line segment $\left[-|G W| \cdot \vec{N}_{G},|G W| \cdot \vec{N}_{G}\right]$, which at each frequency goes in the direction of the normal vector to the curve $\vec{N}_{G}$, and has as magnitude the diameter of $\delta$ (see Fig. 1). This area has as boundaries:

$$
G^{\min }(\mathbf{j} \omega)=G(\mathbf{j} \omega)+\vec{N}_{G} \cdot|G(\mathbf{j} \omega) W(\mathbf{j} \omega)|
$$



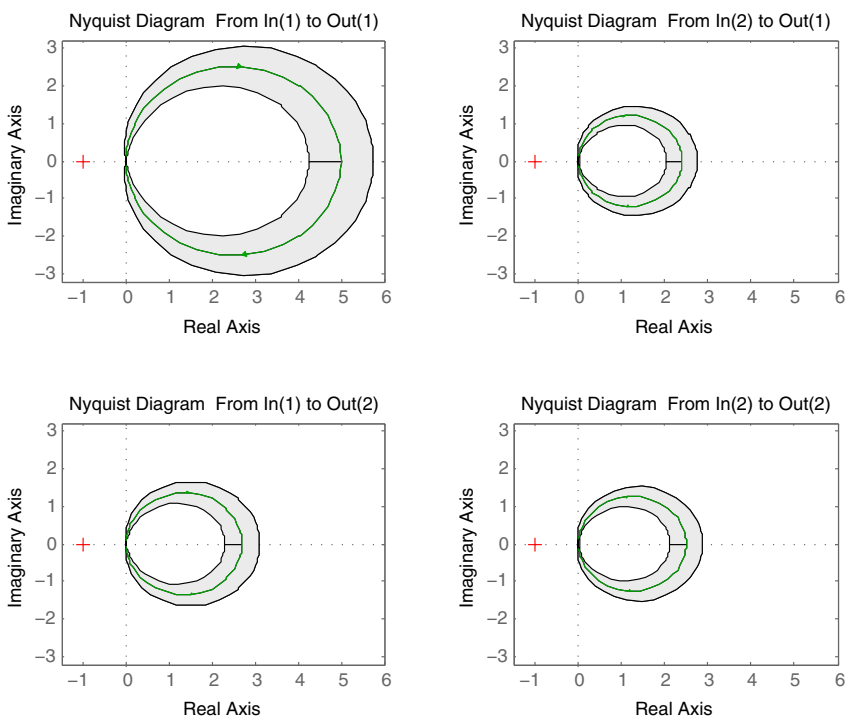

Fig. 2. Uncertain Nyquist diagram for the system in Equation (4) when each input-output channel is affected by a multiplicative uncertainty of $W_{l_{i j}}=$ 0.15 .

$$
G^{\max }(\mathbf{j} \omega)=G(\mathbf{j} \omega)-\vec{N}_{G} \cdot|G(\mathbf{j} \omega) W(\mathbf{j} \omega)|
$$

being the normal vector to the Nyquist diagram:

$$
\vec{N}_{G}(\mathbf{j} \omega)=\frac{G^{\prime}(\mathbf{j} \omega) \times\left(G^{\prime \prime}(\mathbf{j} \omega) \times G^{\prime}(\mathbf{j} \omega)\right)}{\left|G^{\prime}(\mathbf{j} \omega)\right| \cdot\left|G^{\prime}(\mathbf{j} \omega) \times G^{\prime \prime}(\mathbf{j} \omega)\right|}
$$

where $\times$ denotes the vector product, and the differentiation is with respect to $\omega$.

The minimum and maximum values of $\operatorname{tr}(P Q)$ can be approximated from the area enclosed by $G^{\text {min }}$ and $G^{\text {max }}$ respectively.

Example 1. The following model will be used for a robust analysis of interactions based on $\operatorname{tr}\left(P_{j} Q_{i}\right)$.

$$
G(s)=\left(\begin{array}{cc}
\frac{5}{0.5 s+1} & \frac{2.4}{0.25 s+1} \\
\frac{2.7}{0.4 s+1} & \frac{2.5}{0.25 s+1}
\end{array}\right)
$$

Each of the input-output channels is independently perturbed by multiplicative uncertainty with weight $W_{i j}=0.15$. The resulting uncertainty regions in the Nyquist are depicted in Fig. 2. The bounds on $\tilde{\phi}$ have been computed using the integration method described in Appendix B. The resulting bounds on $\tilde{\phi}$ and the nominal value $\tilde{\phi}^{N}$ are:

$$
\tilde{\phi}=\left(\begin{array}{l}
{[4.1435,8.9101][0.9549,2.0533]} \\
{[1.2123,2.5980][1.0359,2.2286]}
\end{array}\right) ; \tilde{\phi}^{N}=\left(\begin{array}{ll}
6.2500 & 1.4400 \\
1.8225 & 1.5625
\end{array}\right)
$$

The sum of the diagonal elements of $\tilde{\phi}$ is, in all cases, larger than the sum of the off-diagonal ones, and therefore this indicator suggests that the best decentralized pairing is the diagonal one. However, if $\tilde{\phi}$ is normalized to obtain the PM, the sum of the main diagonal will vary between 0.53 and 0.84 , which means that such a decentralized structure will consider between $53 \%$ and $84 \%$ of the quantified process dynamics. An upper triangular controller will be the result of discriminating only the subsystem $G_{12}$, and it is the simplest structure which will most likely yield an acceptable performance, since it will consider between $76 \%$ and $94 \%$ of the process dynamics. However, in the worst case for such a triangular structure, the significance of the neglected inputoutput channel is $24 \%$, which is rather large considering that in a system with four equal input-output channels the contribution of each channel is $25 \%$. The final robust decision to be taken is that a full MIMO controller should be used, since no input-output channel can be discriminated considering the variations of $\operatorname{tr}\left(P_{j} Q_{i}\right)$ in the uncertainty set.

\section{B. Exactitude of the approximation}

The discussed approach was proposed in [3], where it was observed that significant errors were committed for systems with large uncertainty and of high order. We derive in the remaining of this section two conditions which have to be satisfied to yield accurate results. If these conditions are not satisfied, the inequality introduced in [2] and later tightened in [3] can alternatively be used with the disadvantage that it is likely to derive in conservative confidence bounds.

1) Condition 1: This approach is based on minimizing or maximizing the radius of the curve at each frequency. Since the center of the curve is located in the direction of the normal vector and at a distance equal to the radius curvature of $G(\mathbf{j} \omega)$, adding or subtracting the value $|G(\mathbf{j} \omega) W(\mathbf{j} \omega)|$ in the direction of the center will locally minimize or maximize the radius of the curve unless $|G(\mathbf{j} \omega) W(\mathbf{j} \omega)|$ is larger than the radius of curvature of $G(\mathbf{j} \omega)$. As a result, the necessary condition for the validity of this approach is that the radius of curvature of $G(\mathbf{j} \omega)$ has to be larger than the radius of the circumference describing the uncertainty set.

2) Condition 2: It was observed in [3] that in some cases the generated boundaries intersect the circles which describe the uncertainty set. This implies that part of the uncertainty set is disregarded. We derive now the reason of this mismatch and describe how significant discrepancies can be identified.

For the generated bounds to include the uncertainty set, it is necessary that they are tangent to the family of curves $\delta$. The tangency condition will then be checked, which is that the tangent vectors to the considered curves at the shared point must go in the same direction.

For representing these tangent vectors, the orthogonal reference frame formed by $\vec{N}_{G}(\mathbf{j} \omega)$ and $\vec{T}_{G}(\mathbf{j} \omega)$ will be used. $\vec{N}_{G}(\mathbf{j} \omega)$ is given by Equation (3), and $\vec{T}_{G}(\mathbf{j} \omega)$ is given by:

$$
\vec{T}_{G}(\mathbf{j} \omega)=G^{\prime}(\mathbf{j} \omega) /\left|G^{\prime}(\mathbf{j} \omega)\right|
$$

The movement of this non-inertial reference frame is given by the Frenet-Serret ([8], [9]) formulae simplified for a planar curve:

$$
\left(\begin{array}{c}
\left(\vec{T}_{G}\right)^{\prime} \\
\left(\vec{N}_{G}\right)^{\prime}
\end{array}\right)=\left|G^{\prime}\right| \cdot\left(\begin{array}{cc}
0 & k_{G} \\
-k_{G} & 0
\end{array}\right)\left(\begin{array}{c}
\vec{T}_{G} \\
\vec{N}_{G}
\end{array}\right)
$$

where $k_{G}(\mathbf{j} \omega)$ is the curvature of $G(\mathbf{j} \omega)$ :

$$
k_{G}(\mathbf{j} \omega)=\left|G^{\prime}(\mathbf{j} \omega) \times G^{\prime \prime}(\mathbf{j} \omega)\right| /\left|G^{\prime}(\mathbf{j} \omega)\right|^{3}
$$

At each frequency $\omega_{k}$, the shared points between $\delta\left(\omega_{k}, \theta\right)$ and the boundaries are obtained in the intersections between the circumference $\delta\left(\omega_{k}, \theta\right)$ and the line determined by $\vec{N}_{G}\left(\mathbf{j} \omega_{k}\right)$. This means that the tangents to $\delta\left(\omega_{k}, \theta\right)$ go 
in the direction of $\vec{T}_{G}\left(\mathbf{j} \omega_{k}\right)$. The direction of the tangent vectors to the boundaries is obtained by differentiating their parametrizations in Equation (2). For the case of $G^{\max }$ and using equations (4) and (5), this yields:

$$
\left(G^{\max }\right)^{\prime}=\left(1+k_{G}\right)\left|G^{\prime}\right| \vec{T}_{G}-|G W|^{\prime} \vec{N}_{G}
$$

which indicates that at each frequency $\omega_{k}$, the boundary $G^{\max }\left(\mathbf{j} \omega_{k}\right)$ is tangent to $\delta\left(\omega_{k}, \theta\right)$ only if the term $\left|G\left(\mathbf{j} \omega_{k}\right) W\left(\mathbf{j} \omega_{k}\right)\right|^{\prime}$ is 0 , meaning that the radius of the circumferences describing the uncertainty set has to remain constant. The angle between both tangent vectors is:

$$
\widehat{T_{G} G^{\text {max }}}=\operatorname{atan}\left(\frac{-|G W|^{\prime}}{\left(1+k_{G}\right) \cdot\left|G^{\prime}\right|}\right)
$$

and in a similar way, for the boundary $G^{\text {min }}$ we obtain:

$$
\widehat{T_{G} G^{m i n}}=\operatorname{atan}\left(\frac{|G W|^{\prime}}{\left(1-k_{G}\right) \cdot\left|G^{\prime}\right|}\right)
$$

These intersection angles are expected to be small for slow variations of the radius of $\delta$ while the Nyquist curve moves in the complex plane. In these cases the error committed in the approximation is found to be negligible (see Fig. 1).

Example 2. The following nominal system and multiplicative uncertainty are considered:

$$
G=\frac{2}{(0.45 s+1)} ; W=\frac{0.312}{(2 s+1)}
$$

It can be observed in Fig. III-B.2(left), that the radius $\delta$ presents fast variations at low frequencies. The intersection angles as a function of the frequency are depicted in Fig. IIIB.2(right). At low frequencies it can be noticed that the intersection angle of $G^{\text {low }}$ is rather large, resulting in a significant part of the circles $\delta$ being neglected. It has been observed that any of these angles being larger than $45^{\circ}$ will most likely derive in a significant part of the circles representing the uncertainty being neglected.

The approximated bounds on $\operatorname{tr}(P Q)$ are given by the interval $[0.7332,1.3850]$. Random simulations were performed by choosing $\Delta$ as a complex number uniformly distributed in the unit circle. For 150 realizations only 1 of them fell out of the approximated bounds.

It can be concluded that the method gives in most cases an accurate approximation, since only abrupt changes in the radius of $\delta$ which persist as the Nyquist curve moves in the complex plane will derive in significant inaccuracies. Besides, multiplicative uncertainty is usually a conservative way of representing uncertainty (see Fig. 1), and therefore it is not significant that the described approach is not capturing a small part of the uncertainty set.

\section{EMPirical Robust estimation of $\operatorname{tr}(P Q)$}

The approach previously described can also be used to obtain an estimation on the bounds of $\operatorname{tr}(P Q)$ from process data. This is done by choosing an appropriate modeling scheme to obtain an estimate for $G(\mathbf{j} \omega)$ at a set of considered frequencies, as well as circular complex regions of uncertainty determined by the variance of the estimators.

The optimal choice of the modeling scheme and the excitation signals is to be decided depending on the process
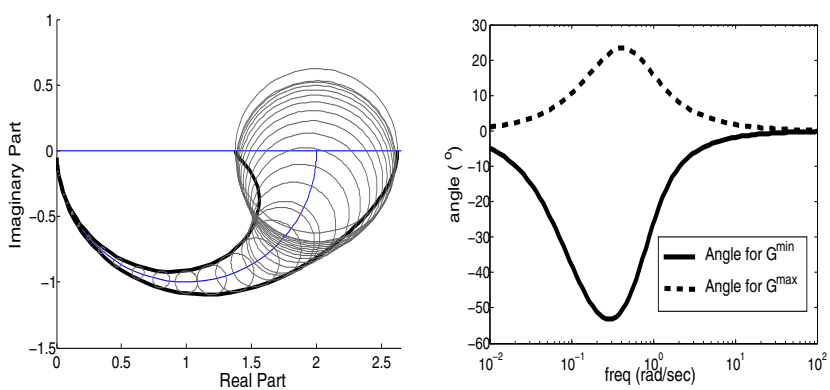

Fig. 3. Uncertainty in the Nyquist diagram for the uncertain system in Equation (10). Left: circles described by multiplicative uncertainty and the approximated boundaries. Right: intersection angles between the approximated boundaries and the circles as function of frequency.

to be modeled and the scenario. However, for a complete illustration of an example, a modeling scheme using the ML estimator for linear multivariable systems is here included.

\section{A. Estimation of the FRF for linear multivariable systems}

Periodic signals have been selected as excitation due to the reduced variability of the obtained FRF estimate compared with i.e. white gaussian noise.

To estimate $G\left(\mathbf{j} \omega_{k}\right)$ for a multivariable system with $n$ inputs and $m$ outputs, $n$ sub-experiments are needed. The Fourier transform of the inputs and outputs for each frequency $\omega_{k}$ will then be collected in the matrices $\mathbf{U}(k) \in$ $\mathcal{C}^{n \times n}$ and $\mathbf{Y}(k) \in \mathcal{C}^{m \times n}$ respectively. In these matrices, the entries $\mathbf{U}_{i j}(k)$ or $\mathbf{Y}_{i j}(k)$ are the frequency content of the $i^{t h}$ input or output at the frequency $\omega_{k}$ and in the $j^{t h}$ subexperiment.

Then the ML estimator $G_{M L}\left(\mathbf{j} \omega_{k}\right)$ is:

$$
G_{M L}\left(\mathbf{j} \omega_{k}\right)=\mathbf{Y}(k) \mathbf{U}^{-1}(k)
$$

In order to guarantee that $\mathbf{U}(k)$ is regular and wellconditioned, the DFT spectrum of one of the input signals will be tailored, and $\mathbf{U}(k)$ will be selected as $\mathbf{U}(k)=$ $U(k) \mathbf{W}$, being $\mathbf{W}$ an orthogonal matrix. I.e. for the case of a $2 \times 2$ system:

$$
\mathbf{W}=\left(\begin{array}{cc}
1 & 1 \\
1 & -1
\end{array}\right)
$$

These orthogonal input signals for TITO systems were introduced in [10] and later considered for an arbitrary input dimension in [11]. They have been designed for attenuating the influence of process noise in the FRF measurements.

In case of having several successive periods of the signals available, different averaging techniques are possible (see [12]), being the one selected here to average the DFT spectrum over the successive periods.

For $P$ measured periods, the estimated model for each period $p$ is denoted as $\left[\hat{G}\left(\mathbf{j} \omega_{k}\right)\right]^{(p)}$. The averaged model is denoted as $\hat{G}\left(\mathbf{j} \omega_{k}\right)$, and the sample variance of the estimator is denoted as $\hat{\sigma}_{i j}^{2}(k)$. They are calculated as:

$$
\begin{array}{r}
\hat{G}\left(\mathbf{j} \omega_{k}\right)=\frac{1}{P} \sum_{p=1}^{P}\left[\hat{G}\left(\mathbf{j} \omega_{k}\right)\right]^{(p)} \\
\hat{\sigma}_{i j}^{2}(k)=\frac{1}{P-1} \sum_{p=1}^{P}\left|\hat{G}_{i j}\left(\mathbf{j} \omega_{k}\right)-\left[\hat{G}_{i j}\left(\mathbf{j} \omega_{k}\right)\right]^{(p)}\right|^{2}
\end{array}
$$




\begin{tabular}{|c|c|c||c|c|c|}
\hline Parameter & Value & Description & Parameter & Value & Description \\
\hline$A_{1}, A_{3}$ & $28 \mathrm{~cm}^{2}$ & Cross section of tanks 1,3 & $k_{1}$ & $3.33 \mathrm{~cm}^{3} / \mathrm{V} \cdot \mathrm{sec}$ & Flow from pump 1 for a voltage unit \\
\hline$A_{2}, A_{4}$ & $32 \mathrm{~cm}^{2}$ & Cross section of tanks 2,4 & $k_{2}$ & $3.35 \mathrm{~cm}^{3} / \mathrm{V} \cdot \mathrm{sec}$ & Flow from pump 1 for a voltage unit \\
\hline$a_{1}, a_{3}$ & $0.071 \mathrm{~cm}^{2}$ & Area of the bottom hole of tanks 1,3 & $\gamma_{1}$ & 0.59 & Flow fraction from pump 1 into tank 1 \\
\hline$a_{2}, a_{4}$ & $0.057 \mathrm{~cm}^{2}$ & Area of the bottom hole of tanks 1,3 & $\gamma_{2}$ & 0.45 & Flow fraction from pump 1 into tank 2 \\
\hline$g$ & $981 \mathrm{~cm} / \mathrm{s}^{2}$ & Gravity acceleration & & & \\
\hline
\end{tabular}

TABLE I

CONSTRUCTION PARAMETERS OF THE QUADRUPLE TANK PROCESS.

\begin{tabular}{|c|c|c|c|c|c|c|}
\hline Variable & $u_{1}^{0}$ & $u_{2}^{0}$ & $h_{1}^{0}$ & $h_{2}^{0}$ & $h_{3}^{0}$ & $h_{4}^{0}$ \\
\hline Value & $3 \mathrm{~V}$ & $3.2 \mathrm{~V}$ & $14.1 \mathrm{~cm}$ & $12.5 \mathrm{~cm}$ & $3.5 \mathrm{~cm}$ & $2.6 \mathrm{~cm}$ \\
\hline
\end{tabular}

TABLE II

SELECTED WORKING POINT FOR THE QUADRUPLE TANK PROCESS.

\section{B. Computing the robust estimation of $\operatorname{tr}(P Q)$}

We will start from an estimation of $G\left(\mathbf{j} \omega_{k}\right)$ and the variance of the estimators at each excited frequency.

For a given confidence $\rho$, circular complex confidence regions for the estimator have a radius of $\sqrt{-\log (1-\rho)} \hat{\sigma}_{i j}(k)$. The confidence $\rho$ will be arbitrary selected with a large value, since in the following, the created confidence regions will be assumed to be reflecting the uncertainty set. The curves enclosing the minimum and maximum areas in the Nyquist diagram can be described as:

$$
\begin{aligned}
G_{i j}^{\min }\left(\mathbf{j} \omega_{k}\right) & =\hat{G}_{i j}\left(\mathbf{j} \omega_{k}\right)+\vec{N}_{G_{i j}}\left(\mathbf{j} \omega_{k}\right) \sqrt{-\log (1-\rho)} \hat{\sigma}_{i j}(k) \\
G_{i j}^{\max }\left(\mathbf{j} \omega_{k}\right) & =\hat{G}_{i j}\left(\mathbf{j} \omega_{k}\right)-\vec{N}_{G_{i j}}\left(\mathbf{j} \omega_{k}\right) \sqrt{-\log (1-\rho)} \hat{\sigma}_{i j}(k)
\end{aligned}
$$

where $\vec{N}_{G i j}\left(\mathbf{j} \omega_{k}\right)$ is the normal vector to the Nyquist curve which was computed using a discrete version of the FrenetSerret frame, and $\rho$ is 0.99 for a region of $99 \%$ confidence.

The area enclosed by the two discretized curves in Equation (13) can be computed using triangulation as described in the appendix. The result is an estimation of $\operatorname{tr}\left(P_{j} Q_{i}\right)$ with uncertainty bounds created by allowing the estimates of the FRF to vary within a circular complex region created from the variance of the estimates.

Example 3. The quadruple-tank system is an interacting system in which two pumps deliver their flow in four interconnected tanks [13]. It is of desire to determine a control structure for controlling the level of the two tanks at the bottom of the construction $\left(h_{1}\right.$ and $\left.h_{2}\right)$ for the working point described in Table 4.2. The linearize model is:

$$
G(s)=\left(\begin{array}{cc}
\frac{\gamma_{1} c_{1}}{1+s T_{1}} & \frac{\left(1-\gamma_{2}\right) c_{1}}{\left(1+s T_{3}\right)\left(1+s T_{1}\right)} \\
\frac{\left(1-\gamma_{1} c_{2}\right.}{\left(1+s T_{4}\right)\left(1+s T_{2}\right)} & \frac{\gamma_{2} c_{2}}{1+s T_{2}}
\end{array}\right)
$$

where $T_{i}=\frac{A_{i}}{a_{i}} \sqrt{2 h_{i}^{0} / g}$ are the time constants of the tanks, $h_{i}^{0}$ is the level of the tank $i$ at the considered working point and $c_{i}=T_{i} k_{i} / A_{i}$. The considered process parameters are summarized in Table 4.1. The inputs $u_{j}$ are the voltage applied to pump $j$ (in Volts), and the outputs $h_{i}$ are the level in $\operatorname{tank} i$ (in $\mathrm{cm}$ ).

Random phase multisine signals [14], [12] were used as excitation for a simulation. The excited frequencies were logarithmically spaced in the interval $\left[f_{0}, f_{m} a x\right]$, with $f_{0}=$ $10^{-4.2} \mathrm{~Hz}$ and $f_{\max }=1585 \cdot f_{0}=10^{-1} \mathrm{~Hz}$. The rms value
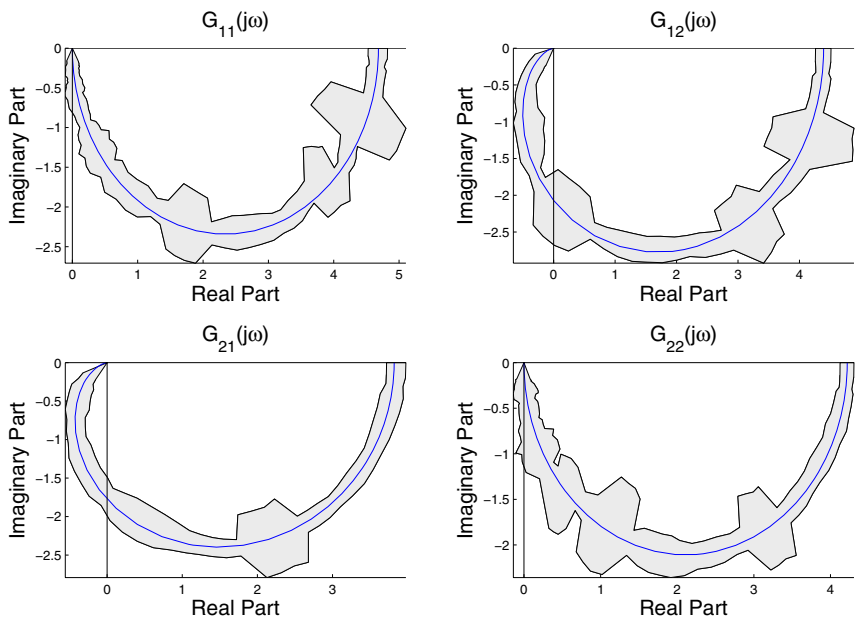

Fig. 4. Smoothed confidence regions in the Nyquist diagram for the magnitude of the estimated nonparametric models for the quadruple tank. The continuous line describes nominal model.

of the excitation was 0.26 . The sampling rate was chosen to be $T s=\left(2 \cdot f_{\max }\right)^{-1}$. The measurements were disturbed with additive white gaussian noise of variance 0.01 . The signals were logged during 5 successive periods, considering 1 period as transient.

The estimated Nyquist curve presents ripples and abrupt variations, deriving in a large uncertainty in the direction of the normal vector. This was solved using the smoothness property of the Nyquist curve. The estimated Nyquist curve and the normal vector were smoothed with a moving average filter, and equations 13 were used to create the uncertainty bounds. These bounds were also smoothed to reduce the uncertainty region, and the result is depicted in Fig. 4. The obtained robust estimation of $\tilde{\phi}$ and the real value are:

$$
\tilde{\phi}=\left(\begin{array}{l}
{[4.1512,6.3226]} \\
{[4.2316,5.7962][3.4430,8.1185]} \\
{[3.4094,5.1468]}
\end{array}\right) ; \tilde{\phi}^{N}=\left(\begin{array}{ll}
5.4852 & 6.9683 \\
5.2242 & 4.4498
\end{array}\right)
$$

\section{CONCLUSIONS}

A method for approximating the bounds on the squared HS norm of uncertain parametric models has been reviewed. The reasons for previously identified inaccuracies have been determined. It has been found that this approach is exact only under specific conditions, but is in most cases a good approximation. Only by models with large uncertainty and/or large number of poles/zeros are likely to present significant discrepancies. If this cases, the inequality introduced in [2] 
and later tightened in [3] can still be used with the disadvantage that it is likely to derive in conservative confidence bounds.

As an application, robust decisions on control structure selection can be performed by inspecting the possible variations of the squared HS norm in the uncertainty set. This is be done by either analyzing an uncertain parametric model, which is traditionally obtained from physical principles and grey-box modeling, or by analyzing an uncertain nonparametric model of the FRF which can be empirically obtained from a tailored experiment.

\section{APPENDIX \\ COMPUTING THE AREA UNDER THE NYQUIST CURVE}

For computing the area enclosed under a Nyquist curve, two methods are here discussed.

\section{A. Computing the area using triangulation}

The curve can be evaluated at a finite number of points, and triangulation techniques can be used to compute the area enclosed by the resulting polygon. For a chosen set of increasing positive frequencies $\omega_{k}=\left\{\omega_{0}, \omega_{1}, \ldots, \omega_{N}\right\}$ the area enclosed by the resulting polygon can be computed as:

$$
\begin{aligned}
& \text { Area }=\left(\operatorname{re}\left(G\left(\mathbf{j} \omega_{N}\right)\right) \cdot i m\left(G\left(\mathbf{j} \omega_{0}\right)\right)-r e\left(G\left(\mathbf{j} \omega_{N}\right)\right) \cdot i m\left(G\left(\mathbf{j} \omega_{N}\right)\right)\right)+ \\
& +\sum_{k=0}^{N-1}\left(r e\left(G\left(\mathbf{j} \omega_{k}\right)\right) \cdot i m\left(G\left(\mathbf{j} \omega_{k+1}\right)\right)-r e\left(G\left(\mathbf{j} \omega_{k+1}\right)\right) \cdot i m\left(G\left(\mathbf{j} \omega_{k}\right)\right)\right)
\end{aligned}
$$

This method is simple to implement and is fast to compute, and many software tools have it in a built in function, like the function polyarea in Matlab. This method it is suitable in the case of having a nonparametric model of the FRF, as derived in Section IV.

A clear disadvantage of this method is that its accuracy depends on the ability of the user in selecting the set of frequencies at which $G(\mathbf{j} \omega)$ is evaluated.

\section{B. Computing the area using numerical integration}

Equations 2 are parametrizations of curves in the complex plane with respect to the parameter $\omega$. The area can therefore be analytically computed by evaluating the following line integral in the complex plane:

$$
\operatorname{Area}(\gamma)=\frac{1}{2} \oint_{\gamma} x d y-y d x
$$

where $\gamma$ denotes the considered curve. However, solving this integral can be tedious, and therefore, a numerical alternative is here discussed. The enclosed area can be computed as:

$$
\operatorname{Area}(\gamma)=\int_{-\infty}^{\infty} \int_{-\infty}^{\infty} n_{v}(x+\mathbf{j} \cdot y) d x d y
$$

where $n_{v}(x+\mathbf{j} \cdot y)$ is the number of times that $\gamma$ winds around the point $x+\mathbf{j} \cdot y$. This integral can numerically be solved using quadrature methods (i.e. the methods implemented in Matlab in the function dblquad). For this purpose, an algorithm computing the winding number of a point in the complex plane needs to be provided to the quadrature method.
Such algorithm is given in [15] as an application of general concepts in geometry applied to the Nyquist diagram. Simplifying results in that paper, the following algorithm can be formulated for computing the winding number for a given point $z_{0}=x_{0}+\mathbf{j} \cdot y_{0}$ :

1.- Denote as $\varrho$ the horizontal half-line which starts at the point $z_{0}$ and goes in the positive direction of the abscissa.

2.- Determine the number and sign of the crossings of $\gamma$ and $\varrho$. A crossing is positive if the cross product of the tangent vectors to the curves $t_{\varrho} \times t_{\gamma}$ is also positive.

3.- The winding number is the number of positive crossings minus the number of negative crossings.

There are two specials cases in this algorithm which have to be clarified. For $\omega \rightarrow \infty$ the Nyquist curve is not smooth. The same situation can occur at $\omega=0$, and therefore, the tangent to the Nyquist curve is not well-defined. The sign of these crossings can be determined by checking the sign of the first nonzero derivative of $\angle G(j \omega)$ for the case of $\omega=0$, and of $\angle G(j / \omega)$ for $\omega \rightarrow \infty$ (see [15]).

\section{ACKNOWLEDGMENTS}

Funding provided by VINNOVA, Hjalmar Lundbohm Research Centre and the participants of the SCOPE consortium, is hereby gratefully acknowledged.

\section{REFERENCES}

[1] M. E. Salgado and A. Conley, "MIMO interaction measure and controller structure selection," International Journal of Control, vol. 77 , no. 4, pp. 367-383, 2004.

[2] B. Moaveni and A. Khaki Sedigh, "Input-output pairing analysis for uncertain multivariable processes," Journal of Process Control, vol. 18, no. 6 , pp. $527-532,2008$.

[3] B. Halvarsson, M. Castaño, and W. Birk, "Uncertainty bounds for gramian-based interaction measures." The 14th WSEAS International Conference on Systems, July 2010.

[4] B. Hanzon, "The area enclosed by the (oriented) Nyquist diagram and the Hilbert-Schmidt-Hankel norm of a linear system," Automatic Control, IEEE Transactions on, vol. 37, no. 6, pp. 835-839, Jun 1992.

[5] S. Skogestad and I. Postlethwaite, Multivariable Feedback Control, 2nd ed. John Wiley and Sons, 2005.

[6] W. Reinelt, A. Garulli, and L. Ljung, "Comparing different approaches to model error modeling in robust identification," Automatica, vol. 38, no. 5, pp. $787-803,2002$.

[7] J. Raisch and B. A. Francis, Modeling Deterministic Uncertainty. CRC Press, 1996, ch. 32 The Control Handbook, p. 551.

[8] F. Frenet, "Sur les courbes à double courbure," pp. 437-447, 1852

[9] J.-A. Serret, "Sur quelques formules relatives à la théorie des courbes à double courbure," pp. 193-207, 1851.

[10] P. Guillaume, R. Pintelon, and J. Schoukens, "Accurate estimation of multivariable frequency response functions," IFAC world conference, pp. 423-428, 1996.

[11] T. Dobrowiecki and J. Schoukens, "Measuring a linear approximation to weakly nonlinear mimo systems," Automatica, vol. 43, no. 10, pp. $1737-1751,2007$.

[12] R. Pintelon and J. Schoukens, System Identification. A frequency domain approach, Piscataway, Ed., 2001.

[13] K. Johansson, "The quadruple-tank process: a multivariable laboratory process with an adjustable zero," Control Systems Technology, IEEE Transactions on, vol. 8, no. 3, pp. 456 -465, May 2000.

[14] J. Schoukens, T. Dobrowiecki, and R. Pintelon, "Parametric and nonparametric identification of linear systems in the presence of nonlinear distortions-a frequency domain approach," Automatic Control, IEEE Transactions on, vol. 43, no. 2, pp. 176 -190, Feb. 1998.

[15] M. Vidyasagar, R. Bertschmann, and C. Sallaberger, "Some simplifications of the graphical nyquist criterion," Automatic Control, IEEE Transactions on, vol. 33, no. 3, pp. 301-305, Mar 1988. 